\title{
Folic acid prevents cardiac dysfunction and reduces myocardial fibrosis in a mouse model of high-fat diet-induced obesity
}

\author{
Wei Li ${ }^{1,2}$, Renqiao Tang ${ }^{1,2}$, Shengrong Ouyang ${ }^{1}$, Feifei Ma ${ }^{1}$, Zhuo Liu ${ }^{1}$ and Jianxin $\mathrm{Wu}^{1,2^{*}}$
}

\begin{abstract}
Background: Folic acid (FA) is an antioxidant that can reduce reactive oxygen species generation and can blunt cardiac dysfunction during ischemia. We hypothesized that FA supplementation prevents cardiac fibrosis and cardiac dysfunction induced by obesity.

Methods: Six-week-old C57BL6/J mice were fed a high-fat diet (HFD), normal diet (ND), or an HFD supplemented with folic acid (FAD) for 14 weeks. Cardiac function was measured using a transthoracic echocardiographic exam. Phenotypic analysis included measurements of body and heart weight, blood glucose and tissue homocysteine (Hcy) content, and heart oxidative stress status.

Results: HFD consumption elevated fasting blood glucose levels and caused obesity and heart enlargement. FA supplementation in HFD-fed mice resulted in reduced fasting blood glucose, heart weight, and heart tissue Hcy content. We also observed a significant cardiac systolic dysfunction when mice were subjected to HFD feeding as indicated by a reduction in the left ventricular ejection fraction and fractional shortening. However, FAD treatment improved cardiac function. FA supplementation protected against cardiac fibrosis induced by HFD. In addition, HFD increased malondialdehyde concentration of the heart tissue and reduced the levels of antioxidant enzyme, glutathione, and catalase. HFD consumption induced myocardial oxidant stress with amelioration by FA treatment.

Conclusion: FA supplementation significantly lowers blood glucose levels and heart tissue Hcy content and reverses cardiac dysfunction induced by HFD in mice. These functional improvements of the heart may be mediated by the alleviation of oxidative stress and myocardial fibrosis.
\end{abstract}

Keywords: High-fat diet, Myocardial fibrosis, Oxidative stress, Folic acid, Cardiac function

\section{Background}

The prevalence of obesity is increasing rapidly worldwide $[1,2]$. Obesity is regarded as an energy balance disorder with inappropriate expansion and dysfunction of the adipose tissue. Obesity causes a prevalence of type 2 diabetes and cardiovascular diseases that cause left ventricular (LV) hypertrophy, stroke, and heart failure [3, 4]. Moreover, accumulated studies have demonstrated that obesity affects cardiac remodeling with structural and functional abnormalities $[5,6]$. However, the underlying mechanisms contributing to

\footnotetext{
* Correspondence: cipbiolab@163.com

${ }^{1}$ Graduate School of Peking Union Medical College, NO. 9, Dongdansantiao, Dongcheng District, Beijing 100730, China

${ }^{2}$ Department of Biochemistry, Capital Institute of Pediatrics, NO. 2, Yabao Road, Chaoyang District, Beijing 100020, China
}

these changes remain unclear because of a complex interplay of hemodynamic, neuroendocrine, and metabolic factors causing cardiac hypertrophy, cellular apoptosis, and interstitial fibrosis [7].

Oxidative stress and inflammation have been considered to be involved in the pathogenesis and progression of many forms of cardiovascular diseases $[8,9]$. Several studies have indicated that oxidative stress plays a pivotal role in the development of obesity-induced cardiomyopathy $[10,11]$. Obesity-related hyperglycemia and metabolic abnormalities cause the production of reactive oxygen species (ROS) in vascular cells and cardiomyocytes [12]. During insulin resistance prior to diabetes onset, ROS accumulation can be found in heart tissue [13], contributing to cardiac dysfunction through lipid 
peroxidation, extracellular matrix remodeling, mitochondrial damage, and alteration in the coupling proteins [14]. In addition, cardiac fibrosis and inflammation are ameliorated by treatment with antioxidants in the diabetic heart $[15,16]$. Therapies aiming to reduce oxidative stress and enhance antioxidant defense have been employed to prevent cardiac dysfunction in cardiovascular diseases [17].

Folic acid (FA) is a B vitamin (vitamin B9) that facilitates the transfer of 1-carbon units in several biosynthetic reactions for two major cellular functions, namely, DNA methylation and the contribution of formyl units to nucleotide synthesis [18]. A meta-analysis of randomized controlled trials has shown that FA supplementation can reduce the risk of cardiovascular diseases [19]. The antioxidant activity is involved in these effects of FA on cardiovascular disorders [20]. Previous studies reported that FA pretreatment blunts myocardial dysfunction during ischemia and ameliorates reperfusion injury and coupled to preservation of high-energy phosphates, reducing ROS generation and ischemia-reperfusion cell death [21].

Hyperhomocysteinemia is associated with a high risk of cardiovascular disease, and dietary folate fortification decreases plasma Hcy levels [22]. The interest in FA for the treatment of cardiovascular disease originates from its critical role in converting Hcy to methionine [23]. FA exhibits an antioxidant effect against ROS and alleviates hyperhomocysteinemia and its associated endothelial dysfunction. Moreover, the anti-inflammatory effect of FA is manifested by a decrease in the levels of some inflammatory mediators in overweight subjects, suggesting a potential therapeutic role of FA in the protection from cardiovascular diseases [24]. Although FA supplementation influences oxidative stress, inflammation, and Hcy metabolism, the direct effect of FA on obesity-related cardiomyopathy has not yet been studied.

In the present study, we hypothesized that FA supplementation in HFD-induced obese mice prevents cardiac dysfunction development by reducing oxidant stress, inflammation, and associated myocardial fibrosis. To test this hypothesis, mice were fed with HFD for 14 weeks in the presence or absence of FA supplementation. Subsequently, mice were measured for weight gain, blood glucose, cardiac function, and heart histology.

\section{Methods}

\section{Animals and experimental design}

Male six-week-old C57BL/6J mice were obtained from Beijing HFK Bioscience Co. Ltd. (Beijing, China) and housed in a temperature-controlled room $\left(23 \pm 2{ }^{\circ} \mathrm{C}\right)$ with $12 \mathrm{~h}$ light-dark cycle. Mice were provided ad libitum access to food and water. Animals were randomly assigned to a normal diet (ND approximately 10\% energy as fat) or a high-fat diet (HFD approximately 60\% energy as fat) or an HFD supplemented with folic acid (FAD, $20 \mu \mathrm{g} / \mathrm{mL}$, Sigma) in drinking water for 14 weeks. The current study used a dose of supplemental FA that is five-times higher than the basal dietary requirements of mice [25]. Body weight was measured weekly. For testing of fasting glucose tolerance, mice were fasted overnight, and then venous blood was drawn by tail clipping. Blood glucose levels were measured with AccuChek glucometers (Roche Applied Science, Penzberg, Germany). Cardiac function, morphology, biochemical parameters, myocardial lipid peroxidation, and oxidative stress profiles were assessed when they reached 20 weeks of age. All the procedures performed were in agreement with the Animal Care and Use Committee at the Capital Institute of Pediatrics (CIP 2016032).

\section{Heart tissue analysis}

The heart tissues were homogenized in a $50 \mathrm{mmol} / \mathrm{L}$ phosphate buffer ( $\mathrm{pH} 7.2)$ containing $0.1 \mathrm{mmol} / \mathrm{L}$ ethylenediamine tetraacetic acid and centrifuged at $5000 \times g$ for $10 \mathrm{~min}$ for subsequent test. The supernatant of heart tissue homogenates was collected for FA and Hcy analysis. Measurements were performed using a FA and Hcy (Cusabio, Wuhan, China) enzyme-linked immunosorbent assay (ELISA) kit according to the manufacturers' instructions.

\section{Echocardiographic examination}

After 14 weeks of feeding, all animals were weighed and evaluated by a transthoracic echocardiographic examination. All the measurements were performed by a technician blinded to the experiments. Mice were anesthetized and analyzed using an animal-specific instrument (VisualSonics Vevo770', Visual Sonics Inc., Toronto, Canada) [26]. LV end diastolic and end-systolic diameters and wall thickness were obtained from Mmode tracings from measurements averaged from six separate cycles. The following structural variables were measured: left ventricular internal diameter at end diastole (LVIDd) and end systole (LVIDs), and LV posterior wall thickness at diastole and systole. Systolic function was assessed based on the LV fractional shortening and LV ejection fraction.

\section{Histological analysis}

After echocardiographic analyses, the mice were euthanized. The heart tissue was also dissected, separated, and weighed. Transverse sections of the LV were fixed in $10 \%$ neutral buffered formalin and paraffin embedded. The five-micron-thick paraffin sections were then stained with hematoxylin and eosin (HE). Masson's trichrome was used for detecting the collagen deposition in the heart tissue. The degree of heart damage was examined blindly using a Leica DMRB/E light microscope 
(Leica Microsystems, Heerbrugg, Switzerland). The heart muscle and vascular smooth muscle were stained pink, whereas the collagen was stained blue.

\section{Oxidative stress analysis}

The heart tissue was used to assess the activity of antioxidant enzymes, superoxide dismutase (SOD) and catalase (CAT), cellular content of antioxidant glutathione (GSH), and lipid peroxidation [malondialdehyde (MDA)]. The levels of SOD, CAT, and GSH enzyme content were measured using a commercialized assay kit (Beyotime Biotechnology, Beijing, China) according to the manufacturer's instructions. Protein contents were determined by bicinchoninic acid methods using a commercial kit (Solarbio, Beijing, China). Enzyme activities were normalized to protein concentration in the samples.

\section{Statistical analysis}

All data sets are presented as the mean \pm standard error of the mean (SEM). Comparison between the two groups was performed using unpaired two-tailed Student $t$-test using Prism 6.0 GraphPad software (GraphPad, San Diego, CA, USA). A value of $p<0.05$ is considered statistically significant.

\section{Results}

\section{FA decreased blood glucose and prevented heart} enlargement in HFD mice

To characterize the effects of HFD feeding on phenotype, we examined bodyweight, heart weight, and fasting blood glucose levels. No difference was observed in the mean daily dietary intake among the three groups. Body weight significantly increased after 14 weeks of HFD feeding (Fig. 1a, $p<0.01$ ). FA supplementation decreased HFD-induced weight gain. However, this difference did not achieve statistical significance (Fig. 1a). Furthermore, we found a significant increase in fasting blood glucose levels in HFD compared with ND, and treatment with the FA markedly decreased blood glucose levels in HFD fed mice (Fig. 1b, $p<0.001$ ), but not back to normal level (Fig. 1b, $p<0.01$ ). In addition, the serum insulin levels were 2.5-fold higher in HFD compared with ND mice, however, there were no significant differences in serum insulin levels between FAD and HFD mice (Fig. 1c, $p<0.05$ ). Homeostatic model assessment of insulin resistance revealed significant insulin resistance in HFD fed mice when compared with the ND groups, and FAD treatment improved insulin sensitivity (data not shown). HFD caused a significant increase in heart weight, and FA supplementation reduced heart weight gain in HFD mice (Fig. 1d, $p<0.05$ ). However, LV mass assessment using ultrasonography showed no difference in the LV weight among the three groups (Fig. 1e).

\section{FA supplementation reversed Hcy elevation induced by HFD}

FA deficiency in serum was associated with the risk of cardiovascular diseases [27]. Therefore, we used tissue FA concentrations as an index of methyl donors in heart tissue extracts. No difference was observed in the FA levels between ND and HFD mice. However, the levels of tissue FA were significantly higher in the FAD group compared with the HFD groups (Fig. $2 \mathrm{a}, p<0.05$ ). We measured heart tissue Hcy levels because FA increases the

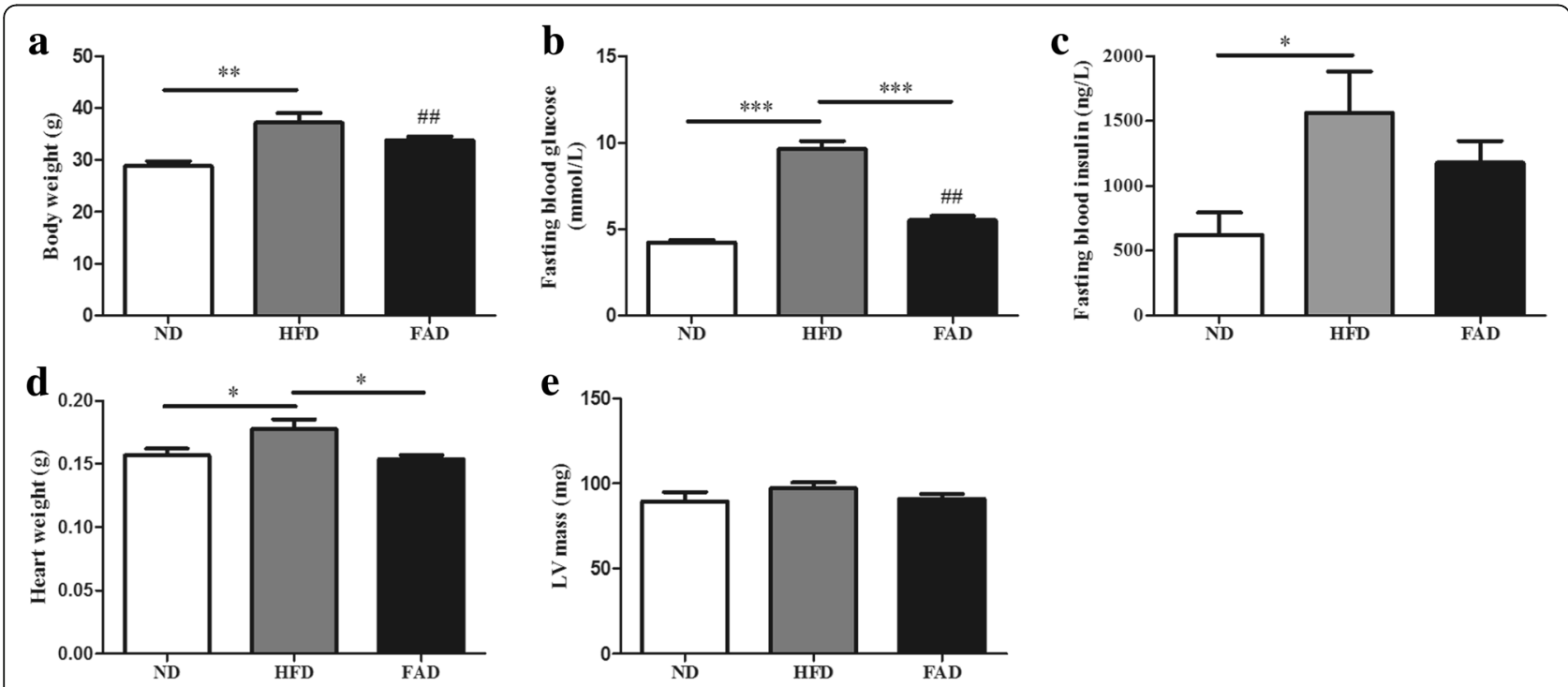

Fig. 1 Effects of folic acid (FA) supplementation on body weight, fasting blood glucose, and organ weight of mice fed with a HFD. a Body weight; (b) fasting blood glucose; (c) fasting blood insulin; (d) heart weight; and (e) LV mass. Values are presented as mean \pm SEM $(n=6)$. ND, normal diet; HFD, high-fat diet; FAD, HFD supplemented with FA; LV, left ventricle. ${ }^{*} p<00.05$; ${ }^{* *} p<0.01$; ${ }^{* *} p<0.001$. \#\#p $<0.01$ versus ND 

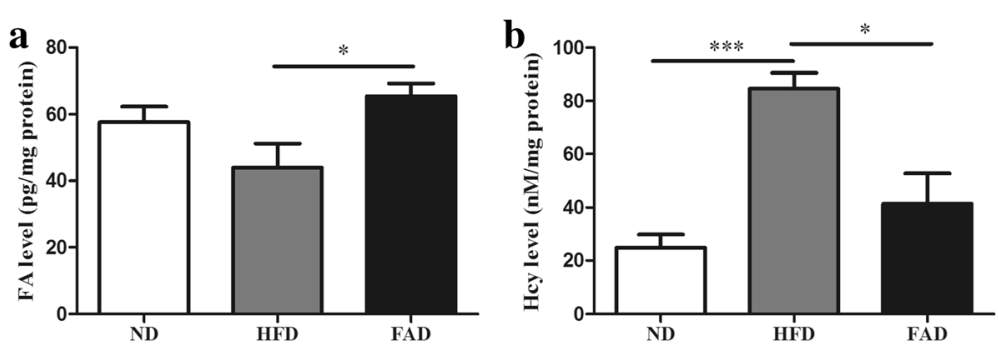

Fig. 2 Levels of FA (a) and Hcy (b) detected in heart tissue by ELISA. Values were presented as mean \pm SEM $(n=6)$. ND, normal diet; HFD, high-fat diet; FAD, HFD supplemented with FA; FA, folic acid; Hcy, homocysteine. ${ }^{*} p<0.05$; ${ }^{* *} p<0.001$

level of 5-methylene tetrahydrofolate reductas, causing an increase in Hcy metabolism [23]. HFD feeding significantly increased Hcy concentrations in heart tissue (Fig. $2 \mathrm{~b}, p<0.001)$. Interestingly, we found that FA supplementation reduced Hcy levels in the heart tissue of HFD mice, but there were no significant differences in Hcy levels between FAD and ND mice (Fig. 2b, $p<0.05$ ).

\section{HFD-induced cardiac dysfunction was improved by FA treatment}

Using 2-D M-mode ultrasonography/echocardiography, we observed the development of cardiac dilatation in mice fed HFD for 14 weeks as determined by LVIDd. LVIDs was significantly high in the HFD groups. LVIDs was significantly decreased in FA treatment mice, but not back to ND mice (Fig. 3a and b, $p<0.05$ ). We also observed a significant cardiac systolic dysfunction when mice were subjected to HFD feeding as indicated by a reduction in LV ejection fraction and fractional shortening (Fig. $3 \mathrm{c}$ and $\mathrm{d}, p<0.01$ ). HFD mice demonstrated that prominent and significant cardiac dilatation and systolic dysfunction compared with ND mice. Although these alterations were relieved by FA treatment, the FAD mice were unable to return to normal level (Fig. $3 \mathrm{c}$ and d). The obesity-induced cardiac dilatation and cardiac dysfunction were improved in mice treated with FA (Fig. $3 \mathrm{c}$ and $\mathrm{d}, p<0.05$ ). However, no difference was found in the LV posterior wall thickness during diastole and systole (Fig. 3e and f).

\section{FA supplementation ameliorated myocardial fibrosis induced by HFD}

To investigate further the cardioprotective effects of FA supplementation, we examined the FA effects on the

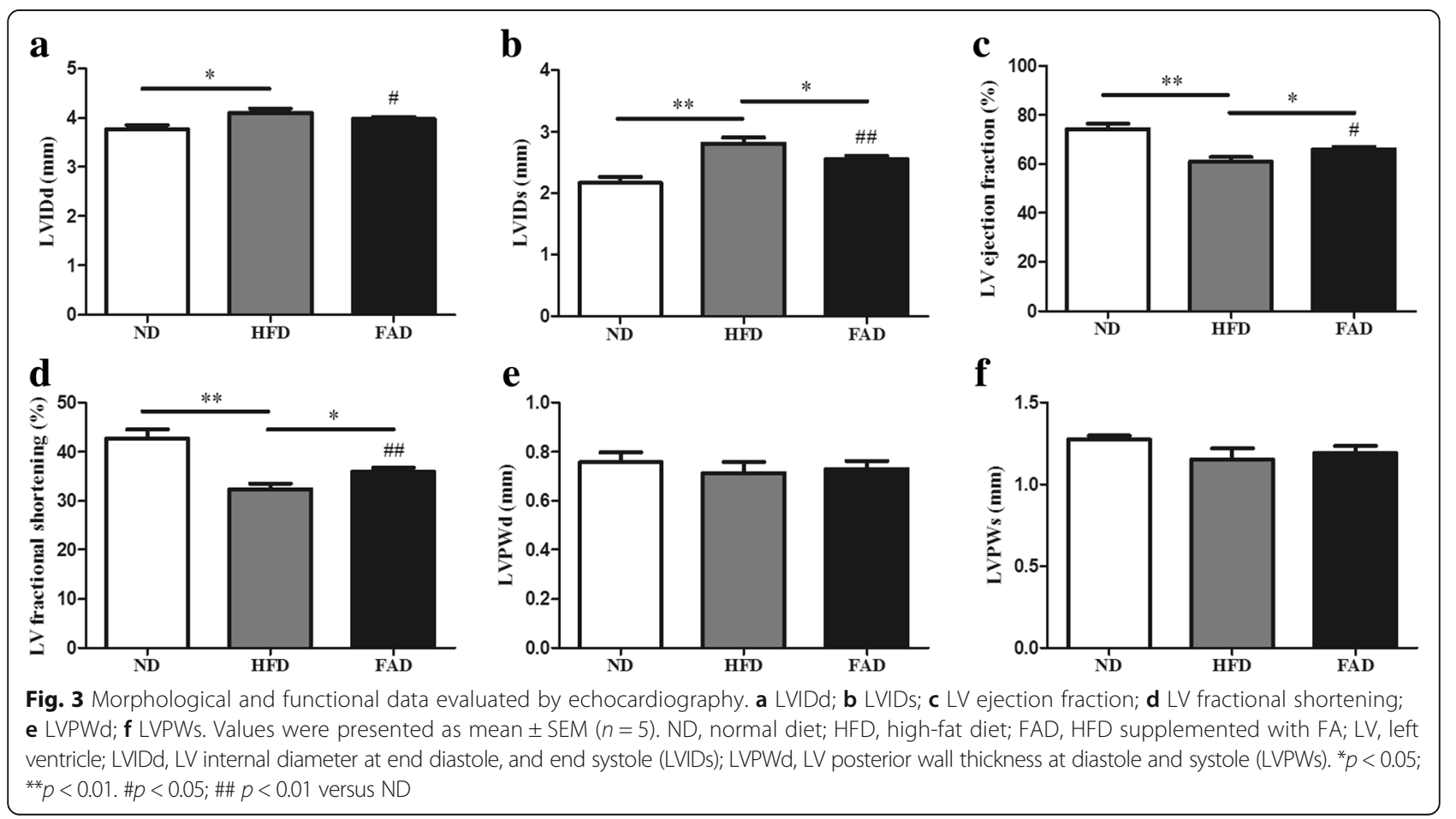


morphology of the heart. HE staining showed that the hearts of HFD-fed mice displayed structural abnormalities and significantly increased fatty degeneration of cardiomyocyte, whereas those alternations of HFD-fed mice were relieved by FA treatment (Fig. 4a). However, no obvious inflammation changes were observed among the three groups. Further staining with Masson's trichrome revealed a significant increase in collagen accumulation and fibrosis in the hearts of HFD-fed mice. Treatment with FA markedly reduced the degree of collagen deposition and fibrosis (Fig. 4b).

\section{FA treatment attenuated myocardial oxidative stress in HFD-fed mice}

Oxidative stress is associated with cardiac dysfunction in models of obesity-related cardiomyopathy [10]. To evaluate the role of FA in oxidative stress, heart redox status of related biomarkers was determined. Analysis of lipid peroxidation by MDA in the myocardium revealed a significant increase in the HFD group. FA supplementation significantly reduced the levels of MDA compared with the HFD group (Fig. 5a, $p<0.05$ ). In addition, HFD consumption obviously caused oxidative stress in the heart tissue as evidenced by significantly reduced the most important antioxidant enzymes GSH and CAT (Fig. 5b and c, $p<0.05)$. However, GSH concentration was increased in mice treated with FA compared with the HFD-fed group but not with the SOD (Fig. 5d). Furthermore, FA treatments restored the concentration of CAT activity to values close to that observed in the ND group (Fig. $5 \mathrm{c}, p<0.05$ ).

\section{Discussion}

The objective of this study was to evaluate the effects of FA supplementation on the HFD-induced obese mice heart and the role of oxidative stress in this scenario. In the current study, mice fed with HFD exhibited greater weight gain compared with ND mice. Glucose and insulin levels were significantly higher in the HFD group, which is indicative of insulin resistance and consistent with the development of type 2 diabetes. FA supplementation reduced fasting blood glucose levels and improved insulin resistance. Furthermore, we demonstrated that FA supplement ameliorated cardiac dysfunction in HFD-induced obese mice. This result was accompanied with decreased cardiac oxidative stress, fibrosis, and tissue Hcy content.

HFD is related to increasing obesity and heart disease, particularly systolic dysfunction, which characterizes early obesity/metabolic cardiomyopathy [28]. Previous experimental evidence have reported the association between obesity and morphological and functional changes in the hearts of both humans and animals [29-31]. Cardiovascular complications in obesity and insulin resistance often manifest as decreased fractional shortening and impaired cardiomyocyte function [32]. In the present study, HFD feeding induced significant cardiac systolic dysfunction, accompanied with significantly increased LVIDd and LVIDs. However, FA supplementation improved LV fractional shortening and LV ejection fraction in HFD mice. This finding indicates that FAD prevents obesity-related cardiac dysfunction in HFD mice.

Multiple pathways have been implicated in the pathology of obesity-related cardiac dysfunction including, LV fibrosis, increased oxidant stress, inflammation, and alterations in collagen content [33]. Studies have repeatedly shown that oxidative stress and myocardial fibrosis are linked to impaired cardiac systolic function in mouse models of obesity and insulin resistance $[34,35]$. In addition, enhanced fatty acid oxidation leads to an overproduction of ROS [36], a byproduct of lipid peroxidation, which plays an important role in increased oxidative stress, as well as diminished energy for myocardial function [37]. Oxidative stress induces several deleterious cardiac alterations including

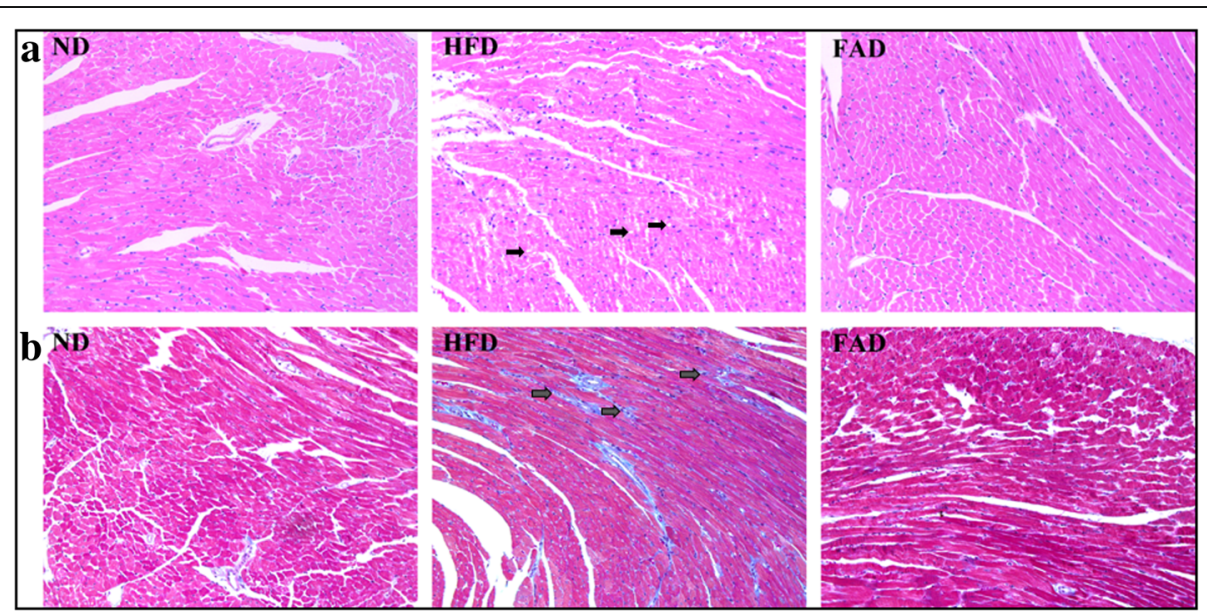

Fig. 4 Heart histology after HE staining (a) and Masson's trichrome staining (b) of sections from each group (original magnification 200x, $n=5$ ). ND, normal diet; HFD, high-fat diet; FAD, HFD supplemented with FA; black arrow, fatty degeneration; gray arrow, collagen accumulation 

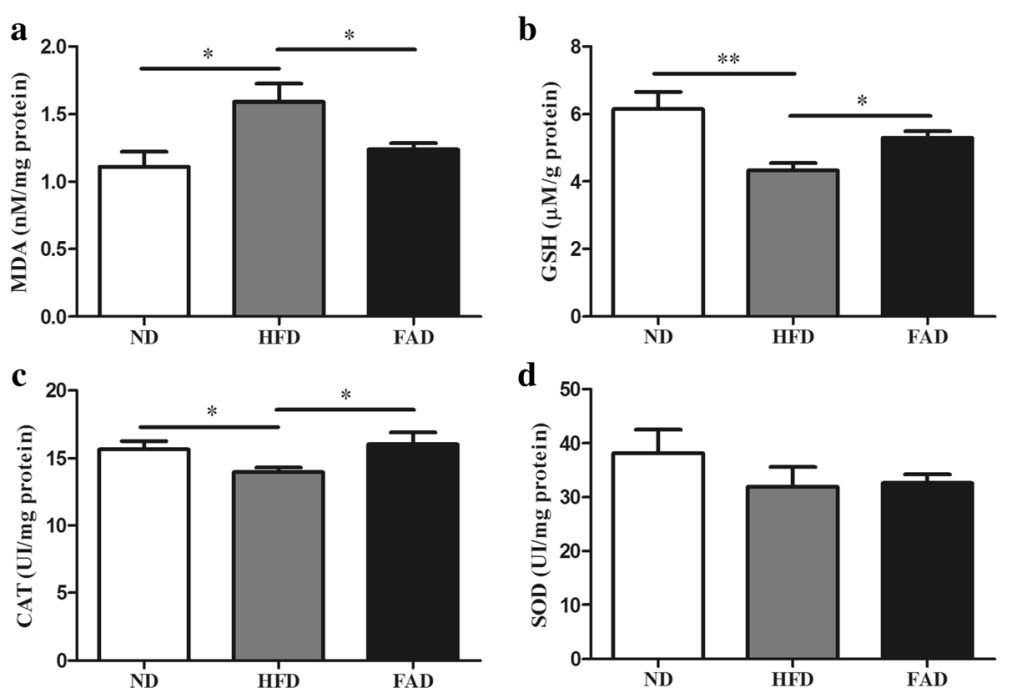

Fig. 5 Effects of FA supplementation on oxidative stress in the heart of mice fed with HFD. a MDA; (b) GSH; (c) CAT; and (d) SOD levels. Values are presented as mean \pm SEM $(n=5-6)$. ND, normal diet; HFD, high-fat diet; FAD, HFD supplemented with FA. ${ }^{*} p<0.05$; ${ }^{* *} p<00.01$

mitochondrial dysfunction, DNA damage, metalloproteinase activation, cellular dysfunction, myocardial fibrosis, and cardiac hypertrophy [38].

Oxidative stress occurs when an imbalance occurs between ROS production and antioxidant systems. Antioxidant enzymes, such as CAT, SOD, and GSH, form the first line of defense against ROS, and the decrease in their activities contribute to the oxidative damage on the tissue [39]. Activities of antioxidant enzymes GSH and CAT decreased in HFD-induced obese mice compared with ND groups and increased in the lipid peroxidation (MDA) in the present study corroborating well with previous studies [40, 41]. FA as a free radical scavenger, if present in physiological concentration, displays potent antioxidant effect, which can protect from free radical damage both directly and via competitive inhibition of xanthine oxidase [42]. The antioxidant activity is thought to be involved in the effects of FA on cardiovascular disorders [20]. Furthermore, the salutary effects of oral FA treatment on endothelial function are partly mediated by an improvement in vascular $\mathrm{BH} 4$ bioavailability, which leads to restoration of eNOS coupling and a reduction of eNOS-derived superoxide production [43]. In the present study, we used a dose of supplemental FA that is five times higher than the basal dietary requirements of mice. We found that the FAD group showed higher CAT and GSH content compared with HFD mice, suggesting an improvement in antioxidant defenses.

Hyperhomocysteinemia is recently recognized as an important independent risk factor for cardiovascular diseases [44]. Previously study suggested that hyperhomocysteinemia caused coronary arteriolar remodeling, myocardial collagen deposition, and diastolic dysfunction in hypertensive rats after 10 weeks of dietary intervention [45]. The detrimental effect of Hcy can be alleviated by FA supplementation with a concurrent reduction of superoxide generation [22]. The metabolism of FA and Hcy is interrelated, and increasing FA intake augments Hcy remethylation, leading to a reduction in its plasma concentration, suggesting that treatment with FA may prevent cardiovascular risk by reducing Hcy [46]. Therefore, the beneficial effect of FA on cardiac function may be confer more credit this interrelation. Folate provides the single-carbon moiety in the synthesis pathway for S-adenosylmethionine, which is the main cellular methyl donor that affects methylation reactions. Supplementation with FA alters global DNA methylation profiles [47]. Thus, FA supplementation may allow post-translational modifications of proteins, which could protect contractile function of the heart. Furthermore, FA has been studied in clinical trials, particularly to test its potential to decrease cardiac dysfunction risk in patients with cardiovascular diseases [48]. Recent study shows that FA mitigated cardiac dysfunction by normalizing the levels of tissue Hcymetabolizing enzymes in myocardial infarction mice [49]. This study emphasizes the importance of Hcy metabolism and FA supplementation in cardiovascular diseases. Previous studies demonstrated that FA ameliorates Hcy-induced oxidative stress [50]. We showed that FA mitigates the cardiac dysfunction in HFD feeding mice. Improvements in oxidant stress and myocardial fibrosis in the heart play a role in this protection. However, further studies are necessary to elucidate the underlying mechanisms. 


\section{Conclusions}

The present study demonstrates that the supplement with FA significantly decreases blood glucose levels, reduces heart tissue Hcy content, and protects against obesity-related cardiac dysfunction induced by HFD in mice. The morphological and functional improvements of the heart may be mediated by the alleviation of lipid peroxidation and myocardial fibrosis. These observations imply that FA supplementation inhibits oxidative stress, and thus, may serve as a potential approach to prevent obesity-related cardiomyopathy.

\section{Abbreviations}

CAT: Catalase; FA: Folic acid; FAD: HFD supplemented with folic acid; GSH: Glutathione; Hcy: Homocysteine; HE: Hematoxylin and eosin; HFD: High fat diet; LV: Left ventricular; MDA: Malondialdehyde; ND: Normal diet; ROS: Reactive oxygen species; SOD: Superoxide dismutase

\section{Acknowledgments}

Not applicable

\section{Funding}

This work was supported by the Beijing Natural Science Foundation (Z140001), the National Basic Research Program of China (2013CB530605), and by the National Natural Science Foundation of China (81400365). This work was also supported in part by the CAMS Central Public Welfare Scientific Research Institute Basal Research Expenses to HW (2016ZX310182-3) and CAMS Initiative for Innovative Medicine (CAMS-I2M).

\section{Availability of data and materials}

All data generated and analysed during the current study are available from the corresponding author on reasonable request.

\section{Authors' contributions}

JW and WL designed the overall study. WL, RT, SO and FM performed the experiments and data analysis. WL and JW was a major contributor in writing the manuscript. All authors read and approved the final manuscript.

\section{Ethics approval}

All the procedures performed were in agreement with the Animal Care and Use Committee at the Capital Institute of Pediatrics (CIP 2016032).

\section{Consent for publication}

Not applicable

\section{Competing interests}

The authors declare that they have no competing interests.

\section{Publisher's Note}

Springer Nature remains neutral with regard to jurisdictional claims in published maps and institutional affiliations.

\section{Received: 15 June 2017 Accepted: 24 October 2017}

Published online: 02 November 2017

\section{References}

1. Prentice AM. The emerging epidemic of obesity in developing countries. Int J Epidemiol. 2006;35:93-9.

2. Flegal KM, Kruszon-Moran D, Carroll MD, Fryar CD, Ogden CL. Trends in obesity among adults in the United States, 2005 to 2014. JAMA. 2016;315:2284-91.

3. Kusminski CM, Bickel PE, Scherer PE. Targeting adipose tissue in the treatment of obesity-associated diabetes. Nat Rev Drug Discov. 2016;15:639-60.

4. Huang PL. eNOS, metabolic syndrome and cardiovascular disease. Trends Endocrinol Metab. 2009;20:295-302.

5. Eschalier R, Rossignol P, Kearney-Schwartz A, Adamopoulos C, Karatzidou K, Fay $R$, et al. Features of cardiac remodeling, associated with blood pressure and fibrosis biomarkers, are frequent in subjects with abdominal obesity. Hypertension. 2014:63:740-6.

6. Jing L, Binkley CM, Suever JD, Umasankar N, Haggerty CM, Rich J, et al. Cardiac remodeling and dysfunction in childhood obesity: a cardiovascular magnetic resonance study. J Cardiovasc Magn Reson. 2016;18:28.

7. Abel ED, Litwin SE, Sweeney G. Cardiac remodeling in obesity. Physiol Rev. 2008:88:389-419.

8. Tousoulis D, Andreou I, Antoniades C, Tentolouris C, Stefanadis C. Role of inflammation and oxidative stress in endothelial progenitor cell function and mobilization: therapeutic implications for cardiovascular diseases. Atherosclerosis. 2008;201:236-47.

9. Lee S, Park Y, Zuidema MY, Hannink M, Zhang C. Effects of interventions on oxidative stress and inflammation of cardiovascular diseases. World J Cardiol. 2011;3:18-24.

10. Illkun O, Boudina S. Cardiac dysfunction and oxidative stress in the metabolic syndrome: an update on antioxidant therapies. Curr Pharm Des. 2013:19:4806-17.

11. Santilli F, Guagnano MT, Vazzana N, La Barba S, Davi G. Oxidative stress drivers and modulators in obesity and cardiovascular disease: from biomarkers to therapeutic approach. Curr Med Chem. 2015;22:582-95.

12. Yan J, Young ME, Cui L, Lopaschuk GD, Liao R, Tian R. Increased glucose uptake and oxidation in mouse hearts prevent high fatty acid oxidation but cause cardiac dysfunction in diet-induced obesity. Circulation. 2009; 119:2818-28.

13. Mellor KM, Ritchie RH, Delbridge LM. Reactive oxygen species and insulinresistant cardiomyopathy. Clin Exp Pharmacol Physiol. 2010;37:222-8.

14. Tsutsui H, Kinugawa S, Matsushima S. Oxidative stress and heart failure. Am J Physiol Heart Circ Physiol. 2011;301:H2181-90.

15. Roslan J, Giribabu N, Karim K, Salleh N. Quercetin ameliorates oxidative stress, inflammation and apoptosis in the heart of streptozotocinnicotinamide-induced adult male diabetic rats. Biomed Pharmacother. 2017:86:570-82

16. Wu H, Li GN, Xie J, Li R, Chen QH, Chen JZ, et al. Resveratrol ameliorates myocardial fibrosis by inhibiting ROS/ERK/TGF-beta/periostin pathway in STZ-induced diabetic mice. BMC Cardiovasc Disord. 2016;16:5.

17. Huynh K, Bernardo BC, McMullen JR, Ritchie RH. Diabetic cardiomyopathy: mechanisms and new treatment strategies targeting antioxidant signaling pathways. Pharmacol Ther. 2014;142:375-415.

18. Salbaum JM, Kappen C. Genetic and epigenomic footprints of folate. Prog Mol Biol Transl Sci. 2012:108:129-58.

19. Li Y, Huang T, Zheng Y, Muka T, Troup J, Hu FB. Folic acid supplementation and the risk of cardiovascular diseases: a meta-analysis of randomized controlled trials. J Am Heart Assoc. 2016:5:e003768.

20. Shirodaria C, Antoniades C, Lee J, Jackson CE, Robson MD, Francis JM, et al. Global improvement of vascular function and redox state with low-dose folic acid: implications for folate therapy in patients with coronary artery disease. Circulation. 2007:115:2262-70.

21. Moens AL, Champion HC, Claeys MJ, Tavazzi B, Kaminski PM, Wolin MS, et al. High-dose folic acid pretreatment blunts cardiac dysfunction during ischemia coupled to maintenance of high-energy phosphates and reduces postreperfusion injury. Circulation. 2008;117:1810-9.

22. Kolling J, Scherer EB, da Cunha AA, da Cunha MJ, Wyse AT. Homocysteine induces oxidative-nitrative stress in heart of rats: prevention by folic acid. Cardiovasc Toxicol. 2011;11:67-73.

23. Zhao M, Wang X, He M, Oin X, Tang G, Huo Y, et al. Homocysteine and stroke risk: modifying effect of Methylenetetrahydrofolate Reductase C677T polymorphism and folic acid intervention. Stroke. 2017:48:1183.

24. Solini A, Santini E, Ferrannini E. Effect of short-term folic acid supplementation on insulin sensitivity and inflammatory markers in overweight subjects. Int J Obes. 2006:30:1197-202.

25. Sie KK, Li J, Ly A, Sohn KJ, Croxford R, Kim YI. Effect of maternal and postweaning folic acid supplementation on global and gene-specific DNA methylation in the liver of the rat offspring. Mol Nutr Food Res. 2013;57:677-85.

26. Sood S, Chelu MG, van Oort RJ, Skapura D, Santonastasi M, Dobrev D, et al. Intracellular calcium leak due to FKBP12.6 deficiency in mice facilitates the inducibility of atrial fibrillation. Heart Rhythm. 2008:5:1047-54

27. Garcia MM, Gueant-Rodriguez RM, Pooya S, Brachet P, Alberto JM, Jeannesson $\mathrm{E}$, et al. Methyl donor deficiency induces cardiomyopathy through altered methylation/acetylation of PGC-1alpha by PRMT1 and SIRT1. J Pathol. 2011;225:324-35. 
28. Dirkx E, Schwenk RW, Glatz JF, Luiken JJ, van Eys GJ. High fat diet induced diabetic cardiomyopathy. Prostaglandins Leukot Essent Fatty Acids. 2011;85:219-25.

29. Alpert MA, Omran J, Mehra A, Ardhanari S. Impact of obesity and weight loss on cardiac performance and morphology in adults. Prog Cardiovasc Dis. 2014:56:391-400.

30. Monte IP, Mangiafico S, Buccheri S, Arcidiacono AA, Lavanco V, Privitera F, et al. Early changes of left ventricular geometry and deformational analysis in obese subjects without cardiovascular risk factors: a three-dimensional and speckle tracking echocardiographic study. Int J Cardiovasc Imaging. 2014;30:1037-47.

31. Saltijeral A, Isla LP, Perez-Rodriguez O, Rueda S, Fernandez-Golfin C, Almeria C, et al. Early myocardial deformation changes associated to isolated obesity: a study based on 3D-wall motion tracking analysis. Obesity (Silver Spring). 2011;19:2268-73.

32. Mahajan R, Lau DH, Sanders P. Impact of obesity on cardiac metabolism, fibrosis, and function. Trends Cardiovasc Med. 2015:25:119-26.

33. Sowers JR, Whaley-Connell A, Hayden MR. The role of overweight and obesity in the Cardiorenal syndrome. Cardiorenal Med. 2011;1:5-12.

34. Zhou X, Ma L, Habibi J, Whaley-Connell A, Hayden MR, Tilmon RD, et al. Nebivolol improves diastolic dysfunction and myocardial remodeling through reductions in oxidative stress in the Zucker obese rat. Hypertension. 2010;55:880-8.

35. Whaley-Connell A, Govindarajan G, Habibi J, Hayden MR, Cooper SA, Wei Y, et al. Angiotensin II-mediated oxidative stress promotes myocardial tissue remodeling in the transgenic (mRen2) 27 Ren2 rat. Am J Physiol Endocrinol Metab. 2007;293:E355-63.

36. Begriche K, Massart J, Robin MA, Bonnet F, Fromenty B. Mitochondrial adaptations and dysfunctions in nonalcoholic fatty liver disease. Hepatology. 2013;58:1497-507.

37. Boudina S, Bugger H, Sena S, O'Neill BT, Zaha VG, Ilkun O, et al. Contribution of impaired myocardial insulin signaling to mitochondrial dysfunction and oxidative stress in the heart. Circulation. 2009;119:1272-83.

38. Kayama Y, Raaz U, Jagger A, Adam M, Schellinger IN, Sakamoto M, et al. Diabetic cardiovascular disease induced by oxidative stress. Int J Mol Sci. 2015;16:25234-63.

39. Li H, Horke S, Forstermann U. Oxidative stress in vascular disease and its pharmacological prevention. Trends Pharmacol Sci. 2013;34:313-9.

40. Xu J, Rong S, Gao H, Chen C, Yang W, Deng Q, et al. A combination of flaxseed oil and Astaxanthin improves hepatic lipid accumulation and reduces oxidative stress in high fat-diet fed rats. Nutrients. 2017;9:271.

41. Xia SF, Le GW, Wang P, Qiu YY, Jiang YY, Tang X. Regressive effect of Myricetin on hepatic Steatosis in mice fed a high-fat diet. Nutrients. 2016;8:799.

42. Stroes ES, van Faassen EE, Yo M, Martasek P, Boer P, Govers R, et al. Folic acid reverts dysfunction of endothelial nitric oxide synthase. Circ Res. 2000 86:1129-34.

43. Antoniades C, Shirodaria C, Warrick N, Cai S, de Bono J. Lee J, et al. 5methyltetrahydrofolate rapidly improves endothelial function and decreases superoxide production in human vessels: effects on vascular tetrahydrobiopterin availability and endothelial nitric oxide synthase coupling. Circulation. 2006;114:1193-201.

44. Mishra PK, Givvimani S, Metreveli N, Tyagi SC. Attenuation of beta2adrenergic receptors and homocysteine metabolic enzymes cause diabetic cardiomyopathy. Biochem Biophys Res Commun. 2010;401:175-81.

45. Joseph J, Washington A, Joseph L, Koehler L, Fink LM, Hauer-Jensen M, et al. Hyperhomocysteinemia leads to adverse cardiac remodeling in hypertensive rats. Am J Physiol Heart Circ Physiol. 2002;283:H2567-74.

46. Carnicer R, Navarro MA, Arbones-Mainar JM, Acin S, Guzman MA, Surra JC, et al. Folic acid supplementation delays atherosclerotic lesion development in apoE-deficient mice. Life Sci. 2007;80:638-43.

47. Langie SA, Achterfeldt S, Gorniak JP, Halley-Hogg KJ, Oxley D, van Schooten FJ, et al. Maternal folate depletion and high-fat feeding from weaning affects DNA methylation and DNA repair in brain of adult offspring. FASEB journal : official publication of the Federation of American Societies for Experimental Biology. 2013;27:3323-34.

48. Huo Y, Li J, Qin X, Huang Y, Wang X, Gottesman RF, et al. Efficacy of folic acid therapy in primary prevention of stroke among adults with hypertension in China: the CSPPT randomized clinical trial. JAMA. 2015;313: 1325-35.

49. Qipshidze N, Tyagi N, Sen U, Givvimani S, Metreveli N, Lominadze D, et al. Folic acid mitigated cardiac dysfunction by normalizing the levels of tissue inhibitor of metalloproteinase and homocysteine-metabolizing enzymes postmyocardial infarction in mice. Am J Physiol Heart Circ Physiol. 2010;299: H1484-93.

50. Miller A, Mujumdar V, Palmer L, Bower JD, Tyagi SC. Reversal of endocardial endothelial dysfunction by folic acid in homocysteinemic hypertensive rats. Am J Hypertens. 2002;15:157-63.

\section{Submit your next manuscript to BioMed Central and we will help you at every step:}

- We accept pre-submission inquiries

- Our selector tool helps you to find the most relevant journal

- We provide round the clock customer support

- Convenient online submission

- Thorough peer review

- Inclusion in PubMed and all major indexing services

- Maximum visibility for your research

Submit your manuscript at www.biomedcentral.com/submit
Biomed Central 